\title{
The increasing incidence of paranasal organizing hematoma: a 20-year experience of 23 cases at a single
} center*

Jong Seung Kim', Jong Seok Oh', Sam Hyun Kwon ${ }^{1,2}$

'Department of Otorhinolaryngology-Head and Neck Surgery, College of Medicine, Chonbuk National University, Jeonju, Korea ${ }^{2}$ Research Institute of Clinical Medicine of Chonbuk National University - Biomedical Research Institute of Chonbuk National University Hospital, Jeonju, Korea
Rhinology 54: 176-182, 2016

DOI:10.4193/Rhino15.158

*Received for publication:

June 1, 2015

Accepted: November 12, 2015

\begin{abstract}
Background and Objectives: Sinonasal organized hematoma is a rare, benign disease that can be locally aggressive and may be mistaken for malignancy. Because of its rarity, the clinical characteristics are not well known. The aim of this study is to investigate the distinguishing features of organized hematoma with an emphasis on incidence change.
\end{abstract}

Materials and Methods: In this retrospective study, we reviewed the records of 23 patients with organized hematoma confirmed histopathologically among 5,378 patients who underwent endoscopic sinus surgery performed by a single surgeon from January 1995 to December 2014 at a tertiary care center. Clinical symptoms, endoscopic photography, computed tomography, and operative findings were reviewed. We also reviewed the relevant literature. Age, sex, site, origin subsite and histopathology were investigated. A statistical review was performed using R 3.1.2 to examine incidence change.

Results: The most common complaint was frequent epistaxis and nasal obstruction (52.1\%). Of the 23 patients, eight were women and 15 were men with an age range of 18 to 75 years. (mean 38.9 years). Nine of these hematomas occurred on the right side and 14 on the left side. The predominant occurrence site was the antrum (65\%), followed by the septum (17.3\%), inferior turbinate (8\%), and ethmoid sinus (8\%). The incidence steadily increased over 20 years.

Conclusion: Investigation of the clinical characteristics and incidence change of organized hematoma can provide useful information. Through analysis of the 23 cases in our study, the age distribution was found to be bimodal and the incidence of organizing hematoma was observed to steadily increase. Clinicians should be aware of these characteristics to avoid misdiagnoses of malignant tumors.

Key words: organizing hematoma, paranasal sinus, endoscopic sinus surgery

\section{Introduction}

Organizing hematoma $(\mathrm{OH})$ is a rare disease entity caused by hemorrhage, fibrosis and neovascularization. The treatment is surgical removal with lavage via endoscopic sinus surgery (ESS), and the recurrence rate is very low. Due to its characteristics of expansion and local destruction, it can be misdiagnosed as a malignancy. Although some studies have reported the clinical characteristics of $\mathrm{OH}$, the long-term incidence change of this entity remains unclear. Therefore, by reviewing 23 cases with a final diagnosis of $\mathrm{OH}$ after endoscopic surgery treated over the past 20 years, we report the incidence change and the analysis of its causes as well as describing the relevant literature. We also review the clinical features, histologic findings, radiologic characteristics, and treatment results of 23 pathologically proven OHs.

\section{Materials and methods}

We retrospectively reviewed the medical records of 23 patients with histopathologically confirmed $\mathrm{OH}$ that underwent surgery at Chonbuk National University Hospital between 1995 and 


\section{4 (Table 1).}

Data on sex, age, incidence change, clinical symptoms, concomitant diseases, origin site, radiologic findings and treatment outcomes were collected and analyzed. The average age was 38.9 years, ranging from 10 to 75 years (SD: 23). The age distribution was bimodal, with two peaks, one among teenagers and one among those over 70 . There were 15 males (65\%) and 8 females (35\%), showing a male predominance of 1.9:1 (Table 1). Fourteen of these lesions occurred on the left side and 9 on the right side. The follow-up period ranged from 2 to 72 months with a mean of 22.8 months (SD: 23.8). Statistical analysis was performed using the Cochran-Armitage trend test in R 3.1.2, and a P-value of less than 0.01 was considered statistically significant.

\section{Results}

Incidence change

Among a total of 5,378 patients who underwent endoscopic surgery from 1995 to 2014 , the incidence rate was $0.4 \%$. On a four-year basis, $\mathrm{OHs}$ were found in 1 of 1,227 patients $(0.08 \%)$ from 1995 to 1998,2 of 1,089 patients $(0.2 \%)$ from 1999 to 2002 , 4 of 1,149 patients $(0.34 \%$ ) from 2003 to 2006,6 of 1,061 patients (0.57\%) from 2007 to 2010 and 9 of 852 patients (1.73\%) from 2011 to 2014 , showing a statistically significant increase $(P<0.01$; Figure 1).

\section{Clinical symptoms}

The most common presenting symptom was nasal obstruction and epistaxis (12 patients, 52\%, respectively), followed by rhinorrhea (6 patients, 26\%), headache (5 patients, $22 \%$ ), postnasal drip and anosmia ( 2 patients, $9 \%$, respectively).

\section{Concomitant diseases}

Among 18 patients (78\%) had underlying diseases; allergic rhinitis (AR) was the most common (10 patients, 43\%), followed by hypertension ( 8 patients, 35\%), dental surgery (6 patients, $50 \%$ ), diabetes mellitus ( 3 patients, $25 \%$ ), trauma ( 2 patients, $17 \%$ ), angina pectoris ( 1 patients, $8 \%$ ), and previous sinus surgery ( 1 patient, $8 \%)$.

\section{Involved site}

The most frequent involved site was the maxillary sinus $(15 / 23=65 \%)$, followed by the nasal septum $(4 / 23=17 \%)$ and ethmoid sinus $(2 / 23=8 \%)$. There were also 2 cases in the inferior turbinate $(2 / 23=8 \%)$. Among the 15 patients with maxillary sinus lesions, 7 were on the right side and 8 were on the left side. There was no significant left or right predominance. All cases were single and unilateral (100\%).

\section{Radiologic findings}

Paranasal CT scan was performed in 19 patients. Soft tissue

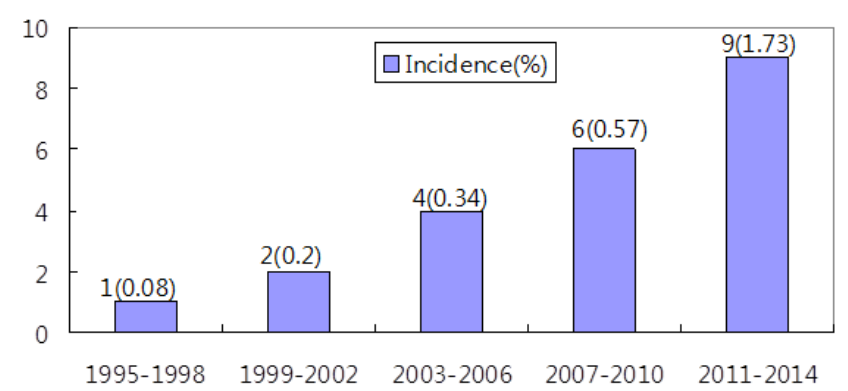

Figure 1. Change in the incidence of organizing hematomas over 20 years using the Cochran-Armitage trend test $(p<0.01)$.

density was seen in the involved sinus in all cases. Eight patients exhibited no evidence of destruction, while 9 patients did have bony destruction on CT scan. The most frequent site of destruction was the medial wall of the maxillary sinus and ethmoid sinus (8/9 cases: $89 \%)$. OHs were heterogeneously enhanced on post-contrast imaging in all cases. Enhanced portions included the antrum, medial wall and center of the maxillary sinus. In two cases, the site of destruction was the anterior end of the inferior turbinate (Figure 2). Four cases showed a heterogeneous enhanced septal mass.

Paranasal magnetic resonance imaging (MRI) was performed in 3 cases. T1-weighted imaging displayed decreased signal intensity with heterogeneous high signal intensity. T2-weighted imaging showed high signal intensity with a multifocal dark signal focus in the center of the mass. The margin, which corresponded to fibrosis, had low signal intensity on T2-weighted imaging. There were heterogeneously strongly enhanced lesions in the center of the mass in all 3 cases (Figure 3). The enhancement pattern was irregular and frond-like with a conglomerated shape that was separated from the normal surrounding tissue.

\section{Preoperative findings}

Preoperative diagnosis of $\mathrm{OH}$ s was generally incorrect. Initial diagnoses included hemangioma, angiofibroma, mucocele, nasal polyp and sinonasal malignancy. Nasal endoscopy indicated that the color of the $\mathrm{OH}$ was usually white $(13 / 23)$ or red $(3 / 23)$. $\mathrm{OH}$ s were sometimes surrounded by polyps, and thus were often misdiagnosed as a polyp.

\section{Treatment outcome}

Endoscopic sinus surgery (ESS) was performed in all cases. Lesions of the anterior and lateral surface of the maxillary sinus required an additional procedure such as a canine fossa approach (21.7\%). The masses were successfully removed via ESS in all cases, but were difficult to eliminate en bloc in a few cases due to fragility and limited exposure. There was no recurrence with a mean follow-up of 22.8 months. There were no residual symptoms or surgical complications. 
Table 1. Nasal obstruction evaluated with objective and subjective methods at different pediatric age ranges. Significance was calculated with ANOVA.

\begin{tabular}{|c|c|c|c|c|c|c|c|c|}
\hline Case & Op year & Age/Sex & Site(sinus) & SD & P. Hx & $\begin{array}{l}\text { Endocopic } \\
\text { finding }\end{array}$ & Op & $\begin{array}{l}\text { Initial } \\
\text { Diagnosis }\end{array}$ \\
\hline 1 & 1998 & $18 / \mathrm{F}$ & $\mathrm{R}(\mathrm{M})$ & - & - & polypoid & $\mathrm{ESS}+\mathrm{CFA}$ & CS, NP \\
\hline 2 & 1999 & $18 / \mathrm{M}$ & $\mathrm{R}(\mathrm{M})$ & $\mathrm{R}$ & AR & W, Ir & $\mathrm{ESS}+\mathrm{CFA}$ & JVA \\
\hline 3 & 2002 & $18 / \mathrm{M}$ & $R(M)$ & - & $A R$ & W, Ir & ESS & $\mathrm{OH}, \mathrm{H}$ \\
\hline 4 & 2005 & $73 / \mathrm{M}$ & $L(M)$ & L & $\begin{array}{l}\text { HTN/DM/trauma/Dental Op } \\
\text { (implant) }\end{array}$ & W, Ir & $\mathrm{ESS}+\mathrm{CFA}$ & - \\
\hline 5 & 2005 & $19 / \mathrm{M}$ & $L(M)$ & L & $A R$ & W (dark red) Ir & ESS & $\mathrm{ACP}, \mathrm{OH}$ \\
\hline 6 & 2006 & $50 / \mathrm{M}$ & $\mathrm{R}(\mathrm{M})$ & - & $\begin{array}{c}\text { Gout/Dental Op (endodontic)/ } \\
\text { AR }\end{array}$ & $\mathrm{R}, \mathrm{Ir}$ & ESS & $\mathrm{OH}$ \\
\hline 7 & 2006 & $33 / \mathrm{M}$ & $\mathrm{L}(\mathrm{M})$ & L & AR & W & ESS & $\mathrm{OH}$ \\
\hline 8 & 2007 & $31 / M$ & $L(E)$ & L & - & NS & ESS & $\mathrm{OH}$ \\
\hline 9 & 2007 & $21 / F$ & $R(E)$ & L & $\mathrm{ESS}(\mathrm{R})$ & $\mathrm{R}$ & ESS & JVA \\
\hline 10 & 2010 & $23 / \mathrm{M}$ & $L(M)$ & $\mathrm{R}$ & - & NS & ESS & Clot \\
\hline 11 & 2010 & $19 / F$ & $\mathrm{R}(\mathrm{M})$ & - & $A R$ & NS & ESS & $\mathrm{ACP}$ \\
\hline 12 & 2010 & $10 / \mathrm{M}$ & $\mathrm{L}(\mathrm{M})$ & $\mathrm{R}$ & - & W, Ir & ESS & - \\
\hline 13 & 2010 & $37 / M$ & $R(M)$ & L & $\begin{array}{l}\text { HTN/trauma/Dental } \\
\text { Op(endodontic) }\end{array}$ & - & ESS & AS \\
\hline 14 & 2011 & $68 / F$ & $\mathrm{~L}(\mathrm{Sp})$ & $\mathrm{L}$ & HTN/angina (aspirin) & W, Ir, crust & ESS & $\mathrm{OH}, \mathrm{IP}$ \\
\hline 15 & 2011 & $18 / \mathrm{F}$ & $\mathrm{R}(\mathrm{M})$ & L & $\begin{array}{c}\text { ESS(R)/Dental Op(endodontic)/ } \\
\text { AR }\end{array}$ & W, Ir & $\mathrm{ESS}+\mathrm{CFA}$ & NP \\
\hline 16 & 2011 & $75 / F$ & $\mathrm{R}(\mathrm{IT})$ & $\mathrm{R}$ & $\begin{array}{l}\text { HTN/DM/cervical Ca/Dental Op } \\
\text { (implant) }\end{array}$ & mass & ESS & $\mathrm{OH}$ \\
\hline 17 & 2012 & $71 / F$ & $L(M)$ & $\mathrm{R}$ & HTN/Dental Op (implant) & $\begin{array}{l}\text { pinkish ex- } \\
\text { pansile mass }\end{array}$ & ESS & - \\
\hline 18 & 2013 & $56 / \mathrm{M}$ & $\mathrm{L}(\mathrm{IT})$ & $\mathrm{L}$ & HTN/DM/AR & W, Ir, polyp & ESS & - \\
\hline 19 & 2013 & $25 / \mathrm{M}$ & $L(M)$ & $\mathrm{R}$ & $A R$ & $\mathrm{R}$, Ir,polyp & ESS & NP \\
\hline 20 & 2014 & $72 / F$ & $L(S p)$ & L & HTN & W, polyp & ESS & $\mathrm{H}$ \\
\hline 21 & 2014 & $73 / \mathrm{M}$ & $\mathrm{L}(\mathrm{Sp})$ & L & HTN & W, polyp & ESS & $\mathrm{H}$ \\
\hline 22 & 2014 & $47 / M$ & $\mathrm{~L}(\mathrm{Sp})$ & L & - & W, Ir, polyp & ESS & $\mathrm{H}, \mathrm{NP}$ \\
\hline 23 & 2014 & 19/M & $L(M)$ & $\mathrm{R}$ & Local nasal biopsy/AR & $\begin{array}{l}\text { W, Ir, yellow } \\
\text { polyp }\end{array}$ & $\mathrm{ESS}+\mathrm{CFA}$ & $\mathrm{OH}$ \\
\hline
\end{tabular}

(Abbreviations : M; male, F; female, R; right, L; left, M; maxillary sinus, E; ethmoid sinus, Sp; septum, IT; inferior turbinate, SD; septal deviation, P. Hx; past history, AR: allergic rhinitis, HTN; hypertension, DM; diabetes mellitus, Op; operation, ESS; endoscopic sinus surgery, Ca; cancer, W; whitish, R; reddish, Ir; irregular surfaced, CFA; canine fossa approach, H; hemangioma, CS; chronic sinusitis, NP; nasal polyp, JVA; juvenile angiofibroma, ACP; antrochoanal polyp, AS; acute sinusitis, IP; inverted papilloma).

\section{Discussion}

$\mathrm{OH}$ is an extremely rare disease first reported by Tadokoro in $1917^{(1)}$. He described it as a hemorrhagic, non-neoplastic mass causing mucosal swelling and bony destruction. This disease has had many names, including "organizing hematoma," "organized hematoma," "hematoma of the maxillary sinus," "hemorrhagic pseudotumor," and "maxillary sinus hematoma" (2-5).
$\mathrm{OH}$ presents much like other masses in the maxillary sinus or other spaces. It involves accumulated hemorrhage in the center and fibrosis along the periphery. If the hemorrhage continues slowly, the lesion grows slowly, compressing adjacent structures, and eventually destroying bony structures. The number of patients whose imaging revealed bony destruction of the medial maxilla was $9 / 19$ (47\%) in this study. Some authors have repor- 


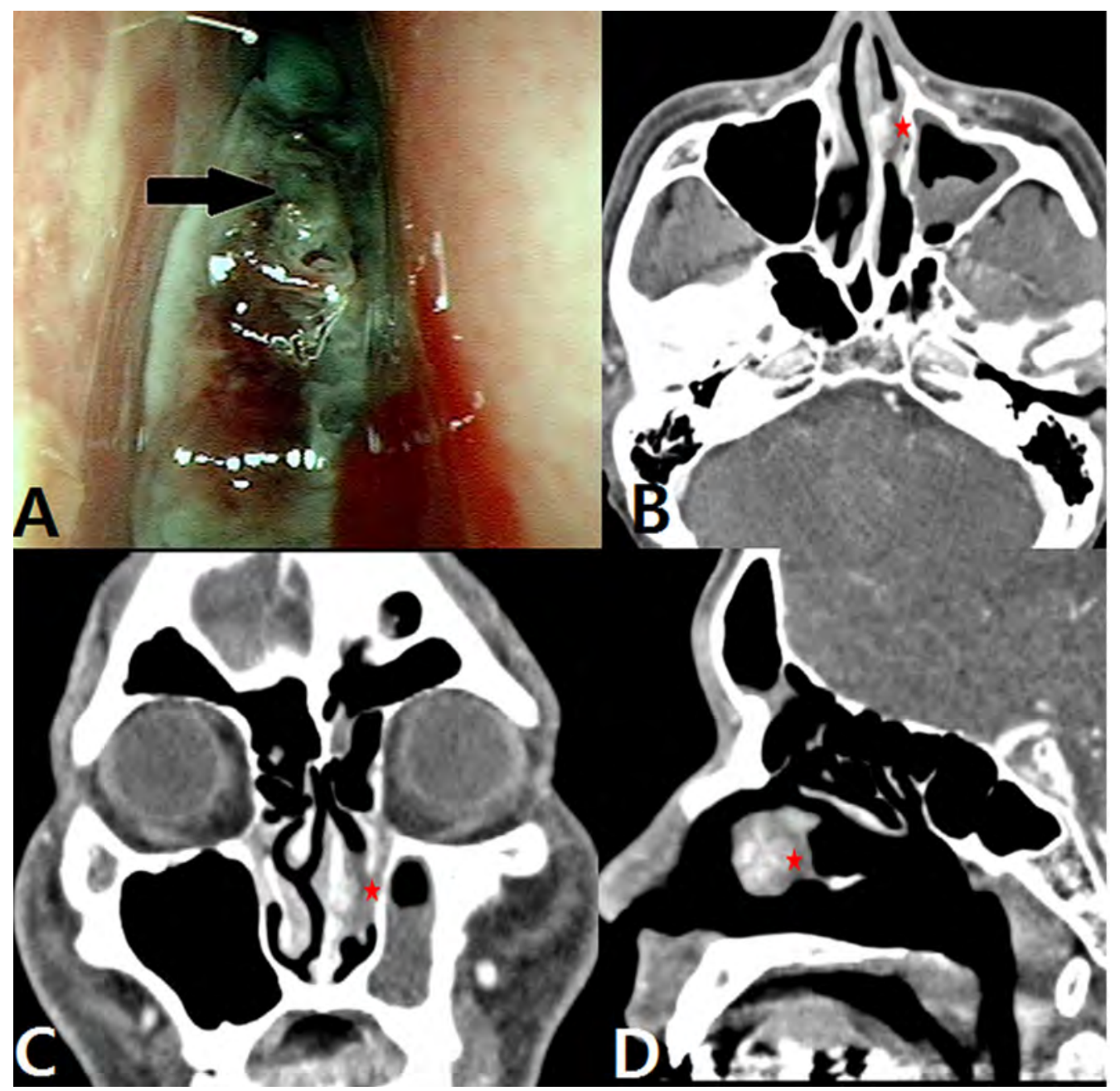

Figure 2. Nasal endoscopy and paranasal CT of case 18. A. A whitish mass with an irregular surface (black arrow) that originated from the inferior turbinate. B. A $1.9 \times 1-\mathrm{cm}$-sized soft mass was noted on axial view post-enhanced CT. Note the erosion of the anterior portion of the left inferior turbinate (red star). C. Coronal view paranasal CT showed a centrally enhanced mass in the inferior turbinate end (red star). D. Sagittal view paranasal CT showed a mass that had eroded the anterosuperior portion of the inferior turbinate (red star).

ted that $39.7 \%$ of patients exhibited bony destruction ${ }^{(6)}$. This characteristic can lead to an incorrect preoperative diagnosis of malignant tumor ${ }^{(7)}$. Another feature that can be mistaken for malignancy is enhancement on CT and MRI. Extravasated blood cells are phagocytosed by the reticulo-endothelial system, resulting in deoxyhemoglobin, methemoglobin, and finally hemichrome and hemosiderin ${ }^{(8)}$. Hemichrome occurs in the center of the $\mathrm{OH}$ in the chronic stage, with iso signal intensity on $\mathrm{T} 1$ and high signal intensity on $\mathrm{T} 2$ imaging. Hemosiderin occurs along the rim of the $\mathrm{OH}$ in the chronic stage, appearing as iso signal intensity on T1 and low signal intensity in T2 imaging ${ }^{(9)}$. On CT and MRI, OHs exhibit heterogeneous mottled enhancement in the central portion and hypointensity along the periphery ${ }^{(6,10)}$. These two findings (bony destruction of the medial maxilla and hypointensity of the periphery on T2-weighted imaging) can differentiate $\mathrm{OH}$ s from malignancies ${ }^{(11)}$. $\mathrm{OH}$ is confirmed on histopathology, which shows hemorrhage, dilated vessels and peripheral fibrosis.

The most frequent symptoms of $\mathrm{OH}$ are nasal obstruction and epistaxis, which are thought result from the mass effect and bleeding tendency of the tumor. Other symptoms exhibited in our study include rhinorrhea, headache, postnasal drip and anosmia. Specific symptom for $\mathrm{OH}$ does not exist, however intermittent epistaxis is worthy of notice ${ }^{(2,12)}$.

Although the pathophysiology of $\mathrm{OH}$ has not been clarified, $\mathrm{OH}$ is thought to develop from accumulation of hemorrhage originating from various causes, such as facial trauma, bleeding diathesis, frequent intranasal bleeding, and vascular injury ${ }^{(10)}$. Our survey reports two cases of trauma and one case of aspirin medication history with $\mathrm{OH}$ (Table 1). Poor ventilation and drainage accelerates the formation of the fibrous capsule and prevents reabsorption of the hematoma and results in neovasculari- 


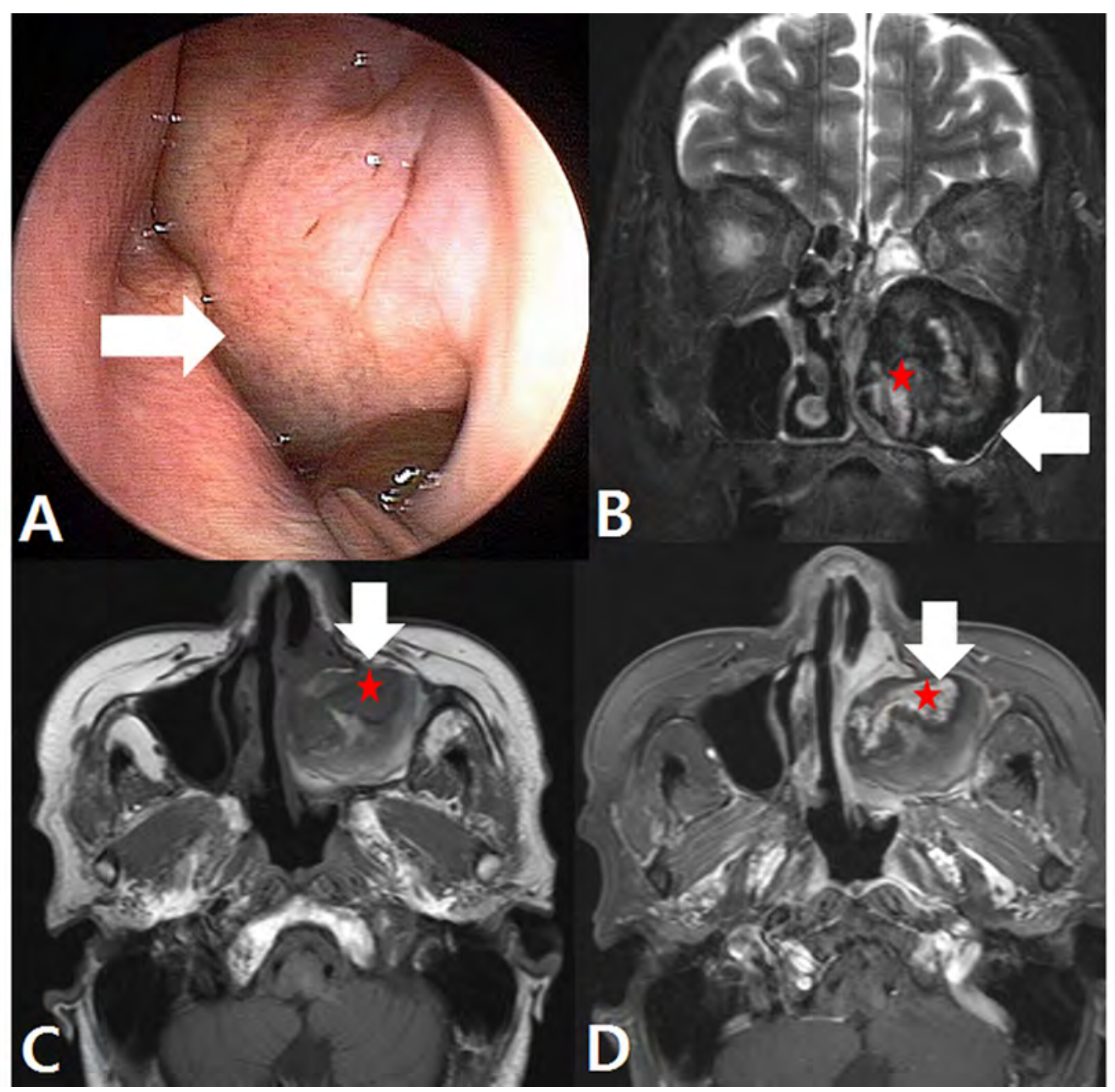

Figure 3. Nasal endoscopy and paranasal CT and MRI of case 17. A. A pinkish expansile mass was noted on the medial surface of the maxilla (white arrow). Dark reddish mucoid contents were extruding. B. Coronal T2-weighted MRI revealed heterogeneous high signal intensity in the center of the mass, in an area of neovascularization and dilated blood vessels (red star). High signal intensity in the periphery (white arrow) shows the intact mucosa of the maxillary sinus. C. Axial T1-weighted MRI showed iso signal intensity (red star) surrounded by homogeneous low signal intensity (white arrow). D. Axial T1-enhanced MRI demonstrated a highly enhanced lesion (red star) with a mix of hypointense, isointense, and hyperintense signals. Note the dark peripheral rim with low signal intensity (white arrow).

zation. The maxillary sinus is the most frequent site of $\mathrm{OH}^{(13)}$. The reason for this is its large size and anti-gravity position of sinus opening, which result in decreased ventilation and negative pressure. Song et al. reported 20 cases of $\mathrm{OH}$ in the maxillary sinus ${ }^{(3)}$.Some authors have suggested that the maxillary sinus is the site of $\mathrm{OH}$ in $90-100 \%$ of cases ${ }^{(6,14)}$. Our study supports that the maxillary sinus is the most frequent site of involvement (15 cases, 65\%). Interestingly, the septum and inferior turbinate were also common sites of occurrence in our study (4 cases and 2 cases, respectively). These 6 cases exhibited septal deviation to the lesion side, which narrows the nasal cavity and causes negative pressure and poor ventilation. Five of these 6 cases also had a history of hypertension, which might be the cause of bleeding. If there is bleeding of the septum or inferior turbinate, which are vessel-rich areas, blood may stay in this narrow space and poor ventilation will accelerate fibrosis and neovascularization. Omura et al. reported one case of $\mathrm{OH}$ located in the right nasal cavity, but the origin was unclear ${ }^{(15)}$. To the best of our knowledge, this is the first report of $\mathrm{OH}$ of the septum or inferior turbinate (Figure 2).

The age distribution of $\mathrm{OH}$ patients shows a bimodal peak in teenagers and those over 70. It is interesting that a high prevalence of $\mathrm{OHs}$ occurred in teenagers in this study compared to other studies ${ }^{(6)}$. Several reports have suggested that $\mathrm{OHs}$ occur at a younger age $(2,3,13,14,16-20)$. Yagisawa et al. also reported a bimodal incidence of $\mathrm{OH}$ by age ${ }^{(1)}$. Half of the cases (3/6) involved teenagers and the rest (3/6) were over 60 years. Our study showed that teenagers have a high prevalence of allergic rhinitis 
compared to those $>20$ years of age (Chi square test, $\mathrm{p}=0.026$ ). The clinical presentation of allergic rhinitis includes mucosal edema and vascular endothelial dilation ${ }^{(21)}$. The increased frequency of AR and epistaxis seems to be associated with a high prevalence of $\mathrm{OH}$ in younger patients.

Precise history taking is very important for the diagnosis of $\mathrm{OH}$. Among 18 patients with underlying disease, hypertension was the most common, followed by dental surgery, diabetes mellitus, nasal trauma, angina pectoris and previous incomplete sinus surgery. There were 3 cases of dental implant history on the lesion side of the maxilla. Dental implantation came into wide use from the late 1990s. There are several cautions when implant on the maxilla than on the mandible, one of which is strongly related to the ossification of maxilla. In situations where the size and shape of the maxillary sinus are relatively larger than the size of the upper jawbone, negative pressure on the maxillary sinus can occur and the success rate of dental implantation may be low without adequate sinus lift or bone graft. Successful dental implantation is affected by surgeon skill level, previous sinusitis history, alveolar bone and ossification of the maxilla, and hemorrhage, hematoma or infection of the maxillary sinus. If these conditions are coincident with impaired mucociliary clearance and poor ventilation in old age, OHs occur in the maxillary sinus and dental surgery is another risk factor of $\mathrm{OH}$, not ever reported ${ }^{(22,23)}$.

There were also 3 cases of ipsilateral endodontic treatment history in our study. This stems from the intimate relationship of the roots of maxillary molars, premolars, and canines with the maxillary sinus ${ }^{(24,25)}$. Endodontic surgery involves obturation of the root canal with a sealant like gutta percha. During this operation, sinusitis may be caused by overinstrumentation, extrusion of chemical agents or sealant material or inappropriate chemical injection. Pathological exposure of the sinus floor can predispose patients to inflammation without appropriate management. Co-occurrence of pathological exposure in the aging population who has decreased mucociliary transport can aggravate the infection and delay hemorrhage absorption. Our study did not objectively prove the correlation between dental implant surgery and $\mathrm{OH}$ incidence; further large-scale studies with detailed medical history taking will be needed.

On the Cochran-Armitage trend test, there was a statistically significant increase in incidence of organizing hematoma. $(P=0.00701)$. Furthermore, a three-fold increase was seen in the second decade compared to the first decade (1995 and 2004); one of the causes of this increase could have been the increasing frequency of dental implantation due to an aging population. Another cause may be the evolution of diagnostic tools like computed tomography and nasal endoscopy.

Finally, the anonymity of this disease is another consideration of this disease. To the best of our knowledge, there was only 1 case report of $\mathrm{OH}$ before the 2000s in the English literature ${ }^{(16)}$. As pre- viously mentioned, $\mathrm{OH}$ has had many different names and there has been no specific distinction between hemorrhagic polyp and $\mathrm{OH}$. The lack of consistency in its name may be a misleading point of diagnosis in the $\mathrm{OHs}$. In fact, OHs were sometimes surrounded by polyps, and thus were often misdiagnosed as a polyp. In case 9 , the patient underwent biopsy at a local clinic. The pathologist reported a 1.2-cm-sized reddish mass with focal hyperplasia of blood vessels, hemorrhage and focal infarction in the surrounding stromal fibrosis. The diagnosis was juvenile angiofibroma, but the pathologist also suspected another disease, because inflammatory cells had infiltrated the lesion and the patient was female. In case 23 , local biopsy results showed that the mass had many macrophages, including hemosiderin, infiltration of inflammatory cells, fibrin and blood vessels, from which it was confirmed to be an inflammatory polyp. Like such cases, correct diagnosis of $\mathrm{OH}$ is even confused in the pathologists. Histologically, fibrosis, hematoma, neovascularization and no evidence of neoplasm are the necessary components of a diagnosis of $\mathrm{OH}^{(12)}$. Careful investigation of all aspects of the mass is important, otherwise otolaryngologists, radiologists and pathologists can misdiagnose this disease.

Although the initial diagnosis is generally incorrect, we extracted some diagnostic criteria suggestive of $\mathrm{OH}: 1$ ) Usually a solitary and unilateral mass; 2 ) The exterior of the mass exhibits a varied fibrous capsule, the color of the capsule is usually whitish, but may be red to dark blue according to the maturation, and the surface of the $\mathrm{OH}$ is usually irregular; 3) Bony erosion can be observed on CT scan due to its expansile nature; 4) Heterogeneous enhancement in the center of the mass and relative low signal intensity in the periphery; 5) The histopathological features of $\mathrm{OH}$ include dilated hemorrhage or hematoma, fibrosis, neovascularization and no evidence of neoplasm. $\mathrm{OH}$ may be confused with nasal polyp and imaging studies like CT or MRI can play an important role in preoperative diagnosis ${ }^{(14)}$.

The treatment of $\mathrm{OH}$ always involves total removal. Although $\mathrm{OHs}$ are usually removed without difficulty, careful and delicate manipulation is essential. In lesions of the septum and inferior turbinate, endoscopic sinus surgery is effective; however, external approaches such as the canine fossa approach are sometimes needed to treat the anterior and lateral aspects of maxillary sinus lesions. To prevent invasive surgical procedures, determination of the clinical aspects of $\mathrm{OH}$, including via imaging study, is needed.

The prognosis of $\mathrm{OH}$ is usually very good and no recurrence was shown in our study. It is important to find and remove the origin of the $\mathrm{OH}$.

\section{Conclusion}

Traditionally, $\mathrm{OHs}$ have been thought to originate from the maxillary sinus. Through 20 years of single-center experience, we report the septum and inferior turbinate as additional 
potential origin sites. Analysis of 23 cases suggested that $\mathrm{OH}$ incidence has steadily increased, with more than a three-fold increase in the second decade (between 2005 and 2014) compared to the first decade (1995-2004). This increase is attributed to early detection through improved diagnostic technologies, increased opportunities for endodontic treatments including dental implantation, and reduced mucociliary transport due to old age. Furthermore, a high frequency of $\mathrm{OH}$ was also shown in the younger age group with allergic rhinitis. Further research on the relationship between the younger age and allergic rhinitis is needed. As the incidence of $\mathrm{OH}$ increases, adequate diagnosis based on the clinical features that distinguish this entity from malignancy on CT or MRI and appropriate treatment with endoscopic sinus surgery are essential.

\section{Acknowledgements}

This paper was supported with funds from the Biomedical Research Institute, Chonbuk National University Hospital.

\section{Authorship contribution}

All authors make substantial contributions to conception and design, and/or acquisition of data, and/or analysis and interpretation of data. All authors participate in drafting the article or revising it critically for important intellectual content. All uthors give final approval of the version to be submitted and any revised version.

\section{Conflict of interest}

None.

\section{References}

1. Yagisawa M, Ishitoya J, Tsukuda M Hematoma-like mass of the maxillary sinus. Acta oto-laryngologica 2006; 126:277-281.

2. Lim M, Lew-Gor S, Beale T, et al. Maxillary sinus haematoma. J Laryngol Otol 2008 122:210-212

3. Song HM, Jang $Y J$, Chung $Y S$, et al Organizing hematoma of the maxillary sinus. Otolaryngol Head Neck Surg. 2007; 136:616-620

4. Suzuki H, Inaba T, Hiraki N, et al. Endoscopic sinus surgery for the treatment of organized hematoma of the maxillary sinus. Kurume Med J. 2008; 55:37-41

5. Unlu HH, Mutlu C, Ayhan S, et al. Organized hematoma of the maxillary sinus mimicking tumor. Auris, nasus, larynx 2001; 28:253-255.

6. Choi SJ, Seo ST, Rha KS, et al. Sinonasal organized Hematoma: Clinical features of seventeen cases and a systematic review. Laryngoscope 2015

7. Yoon TM, Kim JH, Cho YB. Three cases of organized hematoma of the maxillary sinus. Eur Arch Otorhinolaryngol. 2006; 263:823 826.

8. Bradley WG, Jr. MR appearance of hemorrhage in the brain. Radiology 1993; 189:1526.

9. Mattle HP, Edelman RR, Schroth G, Kiefer C. Intracranial hemorrhage. In: EdelmanRR, HesselinkJR, ZlatkinMB, CruesJV (eds): Clinical magnetic resonance imaging, $3 \mathrm{rd}$ ed. Philadelphia: Saunders Elsevier, 2006 pp1287-1345.

10. Kim EY, Kim HJ, Chung SK, et al. Sinonasa organized hematoma: CT and MR imaging findings. AJNR Am J Neuroradiol. 2008; 29:1204-1208.
11. Wu AW, Ting JY, Borgie RC, et al. Diagnostic characteristics of sinonasal organizing hematomas: avoiding misdiagnosis. Int Forum Allergy Rhinol 2013; 3:598-602.

12. Nishiguchi T, Nakamura A, Mochizuki K et al. Expansile organized maxillary sinus hematoma: MR and CT findings and review of literature. AJNR Am J Neuroradiol. 2007 28:1375-1377.

13. Hur J, Kim JK, Byun JS, et al. Imaging characteristics of sinonasal organized hematoma. Acta radiologica 2014

14. Ohta N, Watanabe $T$, Ito $T$, et al. Clinical and pathological characteristics of organized hematoma. Int J Otolaryngol. 2013; 2013:539642.

15. Omura G, Watanabe K, Fujishiro Y, et al. Organized hematoma in the paranasal sinus and nasal cavity--imaging diagnosis and pathological findings. Auris, nasus, larynx 2010; 37:173-177

16. Ozhan S, Arac M, Isik S, et al. Pseudotumor of the maxillary sinus in a patient with von Willebrand's disease. AJR Am J Roentgenol. 1996; 166:950-951.

17. Tabaee A, Kacker A. Hematoma of the maxillary sinus presenting as a mass--a case report and review of literature. Int J Pediatr Otorhinolaryngol. 2002; 65:153-157.

18. Lee BJ, Park HJ, Heo SC. Organized hematoma of the maxillary sinus. Acta oto-laryngologica 2003; 123:869-872

19. Yokoi H, Arakawa A, Matsumoto F, et al Organized hematoma of the maxillary sinus: a clinicopathologic study of 5 cases. Ear Nose Throat J. 2014; 93:E23-26.

20. Lee HK, Smoker WR, Lee BJ, et al. Organized hematoma of the maxillary sinus: CT findings. AJR Am J Roentgenol. 2007;
188:W370-373.

21. Skoner DP. Allergic rhinitis: definition, epidemiology, pathophysiology, detection, and diagnosis. J Allergy Clin Immunol. 2001; 108:S2-8.

22. Incalzi RA, Maini $C L$, Fuso $L$, et al. Effects of aging on mucociliary clearance. Compr Gerontol A. 1989; 3 Suppl:65-68.

23. Ho JC, Chan KN, Hu WH, et al. The effect of aging on nasal mucociliary clearance, beat frequency, and ultrastructure of respiratory cilia. Am J Respir Crit Care Med. 2001; 163:983-988.

24. Hauman $\mathrm{CH}$, Chandler NP, Tong DC. Endodontic implications of the maxillary sinus: a review. Int Endod J. 2002; 35:127141

25. Dodd RB, Dodds RN, Hocomb JB. An endodontically induced maxillary sinusitis. J Endod. 1984; 10:504-506.

Sam Hyun Kwon, MD, PhD

2-20 Keum-Am-Dong

Department of Otorhinolaryngology-

-Head and Neck Surgery

Chonbuk National University Medical

School

Jeonju 561-180

Korea

Tel: +82-63-250-1980

Fax: +82-63-250-1986

E-mail: shkwon@jbnu.ac.kr 